\title{
Population heterogeneity in trajectories of midlife blood pressure
}

\author{
Andrew K Wills ${ }^{1}$, Debbie A Lawlor ${ }^{2}$, Graciela Muniz-Terrera ${ }^{3}$, Fiona Matthews ${ }^{3}$, Rachel \\ Cooper $^{1}$, Arjun K Ghosh ${ }^{1}$, Diana Kuh ${ }^{1}$, and Rebecca Hardy ${ }^{1}$ on behalf of the FALCon Study \\ Team \\ ${ }^{1} \mathrm{MRC}$ Unit for Lifelong Health and Ageing, University College London, UK \\ ${ }^{2}$ MRC Centre for Causal Analyses in Translational Epidemiology, School of Social Medicine, \\ University of Bristol, UK \\ ${ }^{3} \mathrm{MRC}$ Biostatistics Unit, University of Cambridge, UK
}

\begin{abstract}
Background-We investigated whether there are subgroups with different underlying (latent) trajectories of midlife systolic blood pressure (BP), diastolic BP and pulse pressure in a UK cohort.

Methods-Data are from 1840 men and 1819 women with BP measured at age 36, 43 and 53years. We used unconditional growth mixture models to test for the presence of latent trajectory classes. Extracted classes were described in terms of a number of known lifetime risk factors, and linked to the risk of undiagnosed angina (Rose questionnaire) at age 53 years.
\end{abstract}

Results-In both sexes for systolic BP, diastolic BP and pulse pressure, there was a large "normative" class (>90\% of the sample) characterized by gentle annual increases (eg an increase in male systolic BP of $0.9 \mathrm{mmHg} /$ year [ $95 \%$ confidence interval $=0.9$ to1.0]), with a smaller class for whom the rate of increase was high (e.g. an increase in male systolic BP of $3.1 \mathrm{mmHg} / \mathrm{year}$ [2.8 to3.4]). In women there was an additional class for whom BP was high at age 36 and remained high. Persons in the "normative" classes were, on average, heavier at birth and taller at age 7 years, had a lower midlife body mass index, and were less likely to be on antihypertensive medication compared with those in other classes. Among those with no diagnosed cardiovascular disease, those in the classes with more strongly increasing systolic BP and pulse pressure were at greatest risk of angina.

Conclusion-Our study suggests that in midlife the majority of the population have a gentle underlying increase in BP, but that there also exists an important subgroup in whom BP increases much more markedly. These classes may be useful for identifying those most at risk of cardiovascular disease.

Blood pressure (BP) is an important indicator of cardiovascular function, and a strong, modifiable and independent determinant of cardiovascular disease (CVD) and mortality. ${ }^{1,2}$ A person's trajectory of BP during midlife, when the incidence of CVD begins to accelerate, ${ }^{3}$ may be a marker of vascular aging or of an underlying disease process. ${ }^{4}$

Corresponding author: Andrew K Wills, MRC Unit for Lifelong Health and Ageing, University College London, 33 Bedford Place, London, UK. WC1B 5JU. a.wills@nshd.mrc.ac.uk; Tel: INTL + 44 + (0) 207670 5700; Fax: INTL + 44.

SDC: Supplemental digital content is available through direct URL citations in the HTML and PDF versions of this article (www.epidem.com). 
Descriptions of average age-related midlife BP changes in western populations show gentle increases in mean systolic BP from age 30 to 40 years followed by a slightly steeper rise around the $5^{\text {th }}$ decade.$^{5}$ For diastolic BP, more gentle increases are seen up to age $50 .{ }^{6}$ Pulse pressure (the difference between systolic and diastolic BP), which is thought to contain independent information on arterial ageing and CVD risk ${ }^{4,7}$ also increases through midlife. ${ }^{6}$ However, there is considerable variation around these average trends, and examining mean changes in a cohort may conceal unobserved population subgroups that are differentiated by their underlying trajectory. Our recent study of multiple BP trajectories in UK-based cohorts suggested that there may be a period in midlife when blood pressure begins to increase more rapidly in some people. ${ }^{8}$ If population subgroups with different BP trajectories exist, then they may be useful for identifying persons who are at greatest risk of future CVD, and for selecting people who might benefit most from intervention. It is also likely that known BP determinants from across the life course, such as early growth, body mass index (BMI), alcohol intake, activity and socioeconomic circumstances ${ }^{9-13}$ would be associated with these latent trajectories.

One increasingly popular approach for identifying subgroups that share an underlying trajectory is to use latent growth-curve models. A recent study applied this method to a population undergoing treatment for hypertension and found 3 different underlying trajectories. ${ }^{14}$ However, no study has used these methods in a general population cohort. The aim of this paper is to examine whether there are subgroups (classes) in a general population cohort with different underlying trajectories of mid-life systolic BP, diastolic BP and pulse pressure and to explore any links of these various trajectories with a clinical outcome (the prevalence of undiagnosed angina).

\section{Methods}

\section{Study sample}

Data are from the MRC National Survey of Health and Development, a UK birth cohort of 2,547 women and 2,815 men born in one week in March 1946. The cohort has been contacted over 20 times between birth and age 53 years. A full description of the cohort, including initial selection and follow-up has been presented elsewhere; it is in most respects representative of the UK population of a similar age. ${ }^{15,16} \mathrm{BP}$ was measured on three occasions, at age 36, 43 and 53 years. Non-participants at age 36 comprised permanent refusals from earlier surveys $(n=520)$, persons living abroad $(n=644)$ and those who had died $(\mathrm{n}=323)$. Of the 3875 available for contact at age 36 (alive and resident in England, Scotland or Wales and not already a permanent refusal), $86 \%$ participated. Data collections received ethics approval and informed consent was given by respondents.

\section{Blood pressure measurement}

At each assessment, BP was measured twice by a trained nurse in the participant's home, while the survey member was seated and after 5 minutes' rest. We used the second reading where possible or the first if the second was missing ( $<1 \%$ of all observations). At ages 36 and 43 years, a manual random zero sphygmomanometer was used (Hawksley), and at age 53 an automated digital oscillometric sphygmomanometer (Omron HEM-705, Omron Corp., Tokyo, Japan) was used. Corrections for the machine switch in the last wave using published conversion equations made no difference to the results, and so we present the results using the uncorrected values. All readings were taken using an appropriate cuff size for arm circumference. Pulse pressure was calculated as systolic minus diastolic BP. At each assessment, cohort members were asked whether they had taken any prescribed medication or tablets for high BP in the last year. To account for the influence of medication on the BP trajectory and reduce bias caused by treatment effects, a constant of $10 \mathrm{mmHg}$ for systolic 
$\mathrm{BP}$ and $5 \mathrm{mmHg}$ for diastolic BP was added to values that were observed while on treatment. These values were selected on the basis of previously reported estimates for the effect of medication on reducing $\mathrm{BP}^{17,18}$. .

\section{Growth mixture modelling}

Unconditional linear growth mixture models ${ }^{19}$ were used to identify any population substructure in the trajectory of midlife systolic BP, diastolic BP and pulse pressure. Growth mixture models are a combination of a multinomial regression to determine the latent classes and a linear mixed-effects model to estimate BP trajectories in each class. These models are an extension of multilevel models, relaxing the assumption that everyone in the population shares the same mean trajectory, thereby allowing the identification of groups of people who share a similar underlying trajectory (latent class). Under this framework, we compare a sequence of models with increasing numbers of classes to identify any trajectory substructure. A final model is selected that best describes the data in terms of goodness of fit and parsimony. If different subgroups exist, then growth mixture models will provide a better model fit than linear mixed models.

\section{Class characteristics}

Variables that have previously been shown to be associated with BP were used to describe characteristics of people within each latent trajectory class. Birth weight (measured to the nearest $1 / 4$ of a pound and converted to $\mathrm{kg}$ ), considered a marker of the prenatal environment, was measured by an obstetrician or midwife at birth and extracted from health records within a few weeks of birth. Height at age 7 years was measured by health workers and used as a marker of childhood health and nutrition. ${ }^{20}$ Height and weight at age 36 years were measured by nurses and used to calculate body mass index (BMI) (weight $(\mathrm{kg}) /$ height $\left.(\mathrm{m})^{2}\right)$. All height, weight and BMI measures were converted to internally referenced z-scores using the lambda-mu-sigma method. ${ }^{21}$ Socioeconomic position in childhood was described using father's occupation when the cohort member was 4 years old. In adulthood, men's socioeconomic position was represented by their own occupation; women's socioeconomic position was reported by their husband's occupation, except that their own occupation was used where no data existed for the husband or the woman was unmarried. Both socioeconomic-position variables were coded using the Registrar General's classification of occupations and categorized to manual and non-manual classes. Weekly alcohol consumption was measured at age 36 using a 5-day diet diary and 2-day recall (obtained from interviews with a research nurse). Men who drank more than 21 units of alcohol per week and women who drank more than 14 units per week were classified as heavy drinkers (current UK guidelines). At the same age, participants were asked a series of questions on the frequency and duration of participation in sport and recreational activities (using a list of 27 activities such as badminton, swimming, football and jogging) over the last month. Persons who reported no activities were classified as inactive.

\section{Undiagnosed angina}

We identified people with undiagnosed angina at age 53 years (the time of the last blood pressure measurement) using the full Rose questionnaire ${ }^{22} \mathrm{We}$ excluded all those who had been diagnosed by a doctor (as self-reported in questionnaires) with any cardiovascular disease (ischaemic heart disease, coronary thrombosis, stroke or myocardial infarction ([MI]) over their lifetime. Participants with diagnosed CVD are likely to have altered blood pressure, and also may have received treatment or advice that influenced their BP trajectory. Because the remaining participants were unaware of having angina during the period when BP was observed, their BP trajectory is less likely to have been affected by health-related behaviors that might occur in response to a diagnosis of CVD. 


\section{Analyses}

Growth mixture modeling analyses were stratified by sex because the midlife trajectory of $\mathrm{BP}$ is different in men and women ${ }^{5}$ and studies have demonstrated interactions of sex with risk factors for high BP. ${ }^{23}$ To select the model with the best fit, the Bayesian Information Criterion from a series of models with increasing numbers of classes (1 to 8) were compared along with the Lo-Mendell-Rubin likelihood ratio test. A model was assumed to be a better fit if the Bayesian Information Criterion was more than 10 units lower than the more parsimonious model with one fewer class. ${ }^{24}$ As an additional aid to goodness of fit, we examined the model entropy. This is a measure of between-class separation (ie, how well group membership is predicted given the observed data). Model entropy can range from 0 to 1 , with high values indicating good classification. For all models, a linear-growth form was assumed and the variance components were held invariant across classes. The parameter estimates are robust against data missing at random.$^{25}$ Models were estimated with MPlus (v5) using maximum-likelihood methods. Persons were classified into their most probable class using the posterior probabilities estimated from the final growth mixture models. To help interpret each latent class, we summarized the characteristics of persons in each class.

To examine whether any of the latent trajectory classes have predictive value for future cardiovascular disease, we examined the association between the posterior probabilities of class membership with undiagnosed angina at age 53 years, using logistic regression. We pooled men and women for this analysis, as the number with undiagnosed angina was small and a test of a sex interaction with BP class was non-significant. As a comparison with more conventional methodology, we also estimated a linear mixed model for each component of $\mathrm{BP}$, and then extracted the individual fitted random intercepts (which represent blood pressure at age 36), and slopes (change in blood pressure from 36 to 53 years in $\mathrm{mmHg}$ / year). We estimated the association between these intercepts and slopes and undiagnosed angina using logistic regression. Stata (v10.1) was used for this part of the analysis.

To assess the sensitivity of the classes to the choice of constant added to values observed while on antihypertension medication, the analysis was repeated assuming a larger treatment effect (systolic BP, $-15 \mathrm{mmHg}$; diastolic BP, $-10 \mathrm{mmHg}$ ). We further examined of the influence of antihypertensive medication on the latent classes by censoring BP values at the first reported use of medication. More details are in eAppendix 1 (http://links.lww.com).

\section{Results}

Data are from 1840 men and 1819 women with at least one measure of systolic BP and diastolic BP. Sixty-eight percent $(n=2472)$ had complete BP data at age 36,43 and 53 years, $21 \%(n=775)$ had measurements on two occasions and $11 \%(n=412)$ had only one. Figure 1 shows the median pattern of BP change with age. All BP components increased during midlife, and the distributions, particularly the upper tails of systolic BP and pulse pressure, widened with age (also see eAppendix 3, http://links.lww.com). The proportion of people on antihypertensive medication was similar for men and women; overall, $2 \%, 3 \%$ and $15 \%$ were on medication at 36, 43 and 53 years, respectively. Eighty-nine men (6\%) and 53 women $(4 \%)$ reported a previous diagnosis of CVD. Among those with no diagnosis of CVD, 51 (2\%) had angina according to the Rose questionnaire ${ }^{22} ; 35$ of these 51 were women.

\section{Extracted classes}

Figure 2 shows the mean BP trajectories for each latent class in men and women, and Table 1 reports the mean BP at age 36 and slope ( $\mathrm{mmHg} /$ year) between age 36 and 53 years for 
each of these classes. The entropy in all models was good (>0.78) suggesting that the classes distinguished between persons well.

In men, 2 class models had the best fit for systolic BP and diastolic BP according to the BIC (eTable 1, http://links.lww.com). For simplicity we refer to these as the "normative" and "increaser" trajectory classes. The "normative" classes contained more than $90 \%$ of the sample. The mean annual increase in BP in the "increaser" systolic BP and "increaser" diastolic BP class was $3.1 \mathrm{mmHg} /$ year $(95 \% \mathrm{CI}=2.8$ to 3.4 ) and $2.2 \mathrm{mmHg} /$ year (1.9 to 2.5 ), respectively, versus $0.9 \mathrm{mmHg} /$ year and $0.5 \mathrm{mmHg} /$ year in the "normative" systolic and diastolic BP classes respectively. In women, a 3-class model had the best fit for both systolic and diastolic BP. Women had a "normative" and an "increaser" class with qualitatively similar patterns of progression to the men, and an additional class where BP remained high throughout midlife (Table 1). This "high" class was the smallest systolic BP class, containing only $1 \%$ of the sample. By contrast, for diastolic BP the majority of women outside the "normative" class were in the "high" class (3\%), only $1 \%$ were in the "increaser" class.

In both men and women, 3 class models had the best fit for pulse pressure (Figure 2, Table 1 and eTable 1, http://links.lww.com). Both sexes had a "normative" and "increaser" pulse pressure class, characterized by a gentle and steep increase in pressure. There was also a very small group of men $(n=25 ; 1 \%)$ with a high pulse pressure at age 36 that decreased through to age 53 years; we call this the "decreaser" class. Women also had a small third class $(\mathrm{n}=12 ; 1 \%)$ with a pulse pressure that remained "high" from 36 to 53 years.

The majority of men in the pulse pressure "increaser" class were also in the "increaser" systolic-BP trajectory class and "normative" diastolic-BP class (Table 2). Similar results were seen for women. More than a third of men and women in the pulse pressure "increaser" class were classified into the "normative" systolic BP and diastolic BP groups.

\section{Class characteristics}

eTables 8 to 10 (http://links.lww.com) show the mean characteristics of subjects within each latent class. Generally, more characteristics were associated with the systolic BP classes than with the diastolic BP and pulse pressure classes. Persons in the "normative" systolic BP classes were heavier at birth and taller at age 7 and had a lower midlife BMI at age 36. When comparing lifetime BMI and height, patterns of associations with the systolic BP classes were similar but weaker among the pulse pressure "normative" and "increaser" classes. Manual childhood socioeconomic position was associated with the "increaser" systolic BP class in men and women, but not so for diastolic BP classes. Heavy drinking at age 36 was more prevalent in the male systolic BP "increaser" class and female diastolic BP "increaser" class. Women who were inactive at age 36 were more likely to be in the "high" systolic and diastolic BP classes. Use of medication for high blood pressure was most prevalent in the "increaser" classes and highest in the "high" female class ( 70\%).

\section{Class probabilities and undiagnosed angina}

Diastolic BP was omitted from this part of the analysis because of the very small class sizes and inconsistent class structure among men and women. There was no evidence for a sex interaction in the relationship between systolic BP and pulse pressure class probabilities and undiagnosed angina, and so the pooled results are presented (Table 3). The odds of having undiagnosed angina in the systolic BP increaser class were 3.5 times ( $95 \% \mathrm{CI}=1.4$ to 9.0 ) than in the normative class. After adjusting for systolic BP at 36 years, this estimate was attenuated to 2.2 ( $95 \% \mathrm{CI}=0.66$ to 7.5$)$. The results for pulse pressure were similar to those observed in the univariable analysis for systolic BP. Adjusting for pulse pressure at 36 years, 
however, only slightly attenuated the association with the latent classes. The findings from the more standard analysis based on the association between fitted intercepts and slopes from multilevel models and undiagnosed angina showed similar attenuation of the association with the slope after adjustment for BP at baseline.

\section{Sensitivity analysis}

The latent trajectory models were robust to the choice of constant to account for the effects of BP medication (see eAppendix 2.1, http://links.lww.com). However, while the latent classes from models using treatment-censored trajectories were qualitatively similar, there was some discordance in class membership (individuals placed in different classes) compared with the models presented here. Further investigation suggested that this occurred because of a loss of information about the observed trajectory, making the true underlying trajectory more difficult to predict (see eAppendix 2.2 [http://links.lww.com] for more details).

\section{Discussion}

Our study suggests that there are subgroups with different underlying trajectories of midlife blood pressure. In both sexes for systolic BP, diastolic BP and pulse pressure, we identified a large "normative" class containing more than $90 \%$ of the population and characterized by a gentle annual increase in BP, and a smaller "increaser" class from whom the rate of increase was more than three times the rate in the "normative" class. In women there were additional systolic and diastolic BP classes in which BP remained high. As a solitary indicator of a person's blood pressure trajectory, the "increaser" systolic BP or pulse pressure classes were positively associated with undiagnosed angina at the end of the observation period, and there was a very weak suggestion that this trajectory-class result may provide some additional information on risk over and above that of baseline BP.

This is the first study to use growth mixture models to investigate heterogeneity in midlife trajectories of BP in a general population-based study. As this cohort is in most respects representative of the British-born population of the same age, ${ }^{15}$ our findings in terms of class structure may be generalizable to similar western populations. However, it cannot be assumed that similar classes would be obtained with samples from different ethnic groups or from populations with a different distribution of BP determinants. It is also possible that with data extending to older ages, additional subgroups would emerge. Our sensitivity analyses suggested that our findings regarding the existence of trajectory subgroups were robust to how we dealt with observations taken while on BP medication. However, one may expect studies with a greater number of repeat measures to produce more reliable estimates of class membership (see eAppendix 2.2, http://links.lww.com). The independent error components of systolic BP and diastolic BP are combined in the calculation of pulse pressure, which may have increased the likelihood of extracting classes without any substantive meaning or that just reflect measurement error. For example, one may question the meaningfulness of the small "decreaser" and "high" pulse pressure classes found here.

The underlying trajectories in the "normative" BP classes were similar to population averages reported elsewhere. ${ }^{5,6,26}$ However, the trajectories in the other classes were substantially different, with annual increases far greater than the population average. Comparing the characteristics of individuals across classes suggests that a wide range of lifetime circumstances and behaviors were associated with the latent trajectory groups. Low birth weight, ${ }^{27}$ short height at age 7 years,,${ }^{10,28}$ lower lifetime socioeconomic circumstances,${ }^{29}$ higher adult BMI and weight gain, ${ }^{30}$ high alcohol intake, ${ }^{12}$ and low levels of physical activity ${ }^{13}$ — which showed differences among classes-are well established risk factors for high BP, suggesting that the classes do capture groups of persons with differing 
lifetime risk exposures. The stronger association of early-life factors with the systolic BP trajectory classes compared with the diastolic BP classes is also supported by research using more traditional statistical methods, ${ }^{31-33}$ while the similarity between the pulse pressure "increaser" class and systolic BP "increaser" class, in terms of the distribution of life-course characteristics, might reflect the strong influence of systolic BP on pulse pressure trajectory class membership. Although our aim here was to describe the classes, one extension to these unadjusted growth mixture models would be to allow exposures to explain variation in BP within and between classes. This approach could be used to test whether particular risk factors act across the whole distribution of BP, or only on particular groups of people.

The women in the "high" systolic BP and diastolic BP classes were more likely to be on antihypertensive medication and more likely to be inactive compared with women in the other classes, which suggests higher levels of morbidity in those groups. These classes were not present in men, although it is possible that they could emerge at older ages; on average, women show an earlier rise in systolic BP and diastolic BP. ${ }^{6,8}$ It is also possible that different classes would be found if data were available at older ages, particularly for diastolic BP, because around the sixth decade some people experience a levelling-off and decline in blood pressure due to increases in arterial stiffness. ${ }^{4}$

Studies have shown that high blood pressure in midlife is an early manifestation of accelerated arterial aging in later life. ${ }^{4,34}$ Data from the Framingham cohort also show that those with a high BP in early adulthood are more likely to have higher rises in systolic BP and greater decreases in diastolic BP in later life, ${ }^{6}$ or a higher pulse pressure, which may carry an additional risk for cardiovascular mortality. ${ }^{4,7}$ It is unknown whether there is any population substructure in BP among younger adults drawn from similar western populations. If there is population substructure only in midlife but not at younger adult ages, then this would reinforce the midlife period as a transition point when some people begin to follow less-healthy trajectories. Understanding factors that affect this transition would then be important.

While our analysis describes a mean trajectory for each class, there is still substantial variation within these classes such that persons from the "normative" class can still have clinically defined hypertension and two people can have the same current BP but be on a different trajectory. Hence, both absolute current BP and the underlying trajectory may provide independent information about CVD risk. In unadjusted analyses, the "increaser" systolic BP and diastolic BP classes both had higher odds of undiagnosed angina. Further, despite the low statistical power due to the small number of undiagnosed angina cases, our findings tentatively suggest that these classes may have some predictive use independent of a cross-sectional measure of $\mathrm{BP}$, although a stronger assessment of this in relation to future CVD is required. Similar conclusions would, however, be drawn using baseline BP with individual slopes rather than class probabilities. In our setting, the latent trajectory classes may be just a more parsimonious representation of the individual slopes, and if so would not offer additional information on health in later life. It is thus important that careful thought is given to the research question that is being addressed before substituting these models for more conventional approaches to study exposure trajectories. In some cases this choice may depend on whether the research question is person-centered or variable-centered.

It is possible that the classes do not have any substantive meaning beyond providing a better statistical fit of the data against a model with a single underlying population mean. For example, Bauer and Curran ${ }^{35}$ showed in a simulation study that statistical criteria such as the Bayesian information criterion can favor a 2-class model when the data were actually generated from a single skewed and kurtotic distribution. The idea that there is more than one distribution of midlife BP trajectories would also seem to counter the notion of BP as a 
singly distributed continuous variable and polygenic trait. ${ }^{36}$ However, in using unconditional models, our analysis was likely to extract classes that reflect clusters of lifetime BP-influencing exposures and behaviors. While we cannot dismiss the possibility that these trajectories were drawn from a single distribution, one may expect there to be more than one cluster of cardiovascular risk-related behaviors, and in this sense the extracted classes could represent qualitatively different and meaningful groups. As this cohort ages, we will be able to extend these models to allow the classes to predict more concrete cardiovascular endpoints, thereby providing information on the clinical relevance of the analyses we present here.

In conclusion, this study suggests that there are population subgroups with different underlying trajectories of midlife BP. In particular, each component of blood pressure contained a small group of individuals (less than $8 \%$ ) with an underlying trajectory characterized by steeper annual increases. Future studies are required to confirm these groups, examine whether there is population substructure during other periods in life, and assess their value in stratifying risk of future CVD in the general population.

\section{Supplementary Material}

Refer to Web version on PubMed Central for supplementary material.

\section{Acknowledgments}

Other members of the the FALCon (Function and Ageing across the Life Course) study team are Avan Ahie Sayer (MRC Epidemiology Resource Centre, University of Southampton, UK), Eleni Bakra (MRC Biostatistics Unit, University of Cambridge, UK) \& Michaela Benzeval MSc (MRC Social and Public Health Sciences Unit, University of Glasgow, UK). We thank Professor Alun Hughes (Imperial College, UK) for his helpful comments on the manuscript and Dorina Cadar for her help in deriving the alcohol variable. We thank all of the participants of the NSHD for their support and participation in this study, and all staff who were involved in the data collections. The views expressed in this paper are those of the authors and not necessarily those of any funding body or others whose support is acknowledged. The funders had no role in study design, data collection and analysis, decision to publish, or preparation of the manuscript.

Funding sources: A grant from the UK Medical Research Council (MRC) Population health sciences research network (PHSRN29) supported this research and the salary of AKW. DK, RH. GM ((MRC) WBS U. 1052.00.013.00003) and FM (U.1052.00.013.00001) are supported by the UK MRC. RC is receiving support from the HALCyon programme funded by the New Dynamics of Ageing (RES-353-25-0001). The UK MRC and the University of Bristol provide core funding for the MRC Centre of Causal Analyses in Translational Epidemiology where DAL works. The UK MRC has provided funding for the NSHD since 1962.

\section{Reference List}

1. Lewington S, MacMahon S. Blood Pressure, Cholesterol, and Common Causes of Death: a Review. Prospective Studies Collaboration. Am.J.Hypertens. 1999; 12(10 Pt 2):96S-8S. [PubMed: 10555608]

2. Lewington S, Clarke R, Qizilbash N, Peto R, Collins R. Age-Specific Relevance of Usual Blood Pressure to Vascular Mortality: a Meta-Analysis of Individual Data for One Million Adults in 61 Prospective Studies. Lancet. Dec 14; 2002 360(9349):1903-13. [PubMed: 12493255]

3. Health Survey for England 2006. 2008; Volume 1. Cardiovascular disease and risk factors in adults

4. Benetos, Athanase. Does Blood Pressure Control Contribute to a More Successful Aging? Hypertension. Aug 1; 2005 46(2):261-2. [PubMed: 15983236]

5. Bazzano, LA.; Whelton, PK.; He, J. Blood pressure in westernized and isolated populations. In: Lip, GYH.; Hall, JE., editors. Comprehensive Hypertension. Mosby, Elsevier; Philadelphia: 2007. p. 21-30.

6. Franklin, Stanley S.; Gustin; William, IV.; Wong, Nathan D.; Larson, Martin G.; Weber, Michael A.; Kannel, William B.; Levy, Daniel. Hemodynamic Patterns of Age-Related Changes in Blood 
Pressure : The Framingham Heart Study. Circulation. Jul 1; 1997 96(1):308-15. [PubMed: 9236450]

7. Strandberg TE, Pitkala K. What Is the Most Important Component of Blood Pressure: Systolic, Diastolic or Pulse Pressure? Curr.Opin.Nephrol.Hypertens. 2003; 12(3):293-7. [PubMed: 12698068]

8. Wills, Andrew K.; Lawlor, Debbie A.; Matthews, Fiona E.; Bakra, Eleni; Ben Shlomo, Yoav; Benzeval, Michaela; Brunner, Eric; Cooper, Rachel; Kivimaki, Mika; Kuh, Diana; Muniz-Terrera, Graciela; Hardy, Rebecca. Life Course Trajectories of Systolic Blood Pressure Using Longitudinal Data From Eight UK Cohorts. PLoS Med. Jun 14.2011 8(6):e1000440. [PubMed: 21695075]

9. Hardy R, Wadsworth ME, Langenberg C, Kuh D. Birthweight, Childhood Growth, and Blood Pressure at 43 Years in a British Birth Cohort. Int.J Epidemiol. 2004; 33(1):121-9. [PubMed: 15075157]

10. Ben Shlomo Y, McCarthy A, Hughes R, Tilling K, Davies D, Smith GD. Immediate Postnatal Growth Is Associated With Blood Pressure in Young Adulthood: the Barry Caerphilly Growth Study. Hypertension. 2008; 52(4):638-44. [PubMed: 18768401]

11. Chris, Power; Kate, Atherton; Strachan, David P.; Shepherd, Peter; Fuller, Elizabeth; Davis, Adrian; Gibb, Ian; Kumari, Meena; Lowe, Gordon; Macfarlane, Gary J.; Rahi, Jugnoo; Rodgers, Bryan; Stansfeld, Stephen. Life-Course Influences on Health in British Adults: Effects of SocioEconomic Position in Childhood and Adulthood. International Journal of Epidemiology. Jun 1; 2007 36(3):532-9. [PubMed: 17255345]

12. Chen L, Davey Smith G. Harbord RM, Lewis SJ. Alcohol Intake and Blood Pressure: a Systematic Review Implementing a Mendelian Randomization Approach. PLoS.Med. Mar 4.2008 5(3):e52. [PubMed: 18318597]

13. Ekelund U, Franks PW, Sharp S, Brage S, Wareham NJ. Increase in Physical Activity Energy Expenditure Is Associated With Reduced Metabolic Risk Independent of Change in Fatness and Fitness. Diabetes Care. 2007; 30(8):2101-6. [PubMed: 17536069]

14. Ho PM, Magid DJ, Shetterly SM, Olson KL, Peterson PN, Masoudi FA, Rumsfeld JS. Importance of Therapy Intensification and Medication Nonadherence for Blood Pressure Control in Patients With Coronary Disease. Arch.Intern.Med. Feb 11; 2008 168(3):271-6. [PubMed: 18268167]

15. Wadsworth M, Kuh D, Richards M, Hardy R. Cohort Profile: The 1946 National Birth Cohort (MRC National Survey of Health and Development). International Journal of Epidemiology. 2006; 35(1):49-54. [PubMed: 16204333]

16. Wadsworth ME, Mann SL, Rodgers B, Kuh DJ, Hilder WS, Yusuf EJ. Loss and Representativeness in a 43 Year Follow Up of a National Birth Cohort. J.Epidemiol.Community Health. 1992; 46(3):300-4. [PubMed: 1645091]

17. Tobin MD, Sheehan NA, Scurrah KJ, Burton PR. Adjusting for Treatment Effects in Studies of Quantitative Traits: Antihypertensive Therapy and Systolic Blood Pressure. Stat.Med. Oct 15; 2005 24(19):2911-35. [PubMed: 16152135]

18. Wu J, Kraja AT, Oberman A, Lewis CE, Ellison RC, Arnett DK, Heiss G, Lalouel JM, Turner ST, Hunt SC, Province MA, Rao DC. A Summary of the Effects of Antihypertensive Medications on Measured Blood Pressure. Am.J.Hypertens. 2005; 18(7):935-42. [PubMed: 16053990]

19. Muthen B, Shedden K. Finite Mixture Modeling With Mixture Outcomes Using the EM Algorithm. Biometrics. 1999; 55(2):463-9. [PubMed: 11318201]

20. Gunnell, David. Commentary: Can Adult Anthropometry Be Used As a 'Biomarker' for Prenatal and Childhood Exposures? International Journal of Epidemiology. Apr 1; 2002 31(2):390-4. [PubMed: 11980801]

21. Cole TJ. The LMS Method for Constructing Normalized Growth Standards. Eur.J Clin Nutr. 1990; 44(1):45-60. [PubMed: 2354692]

22. Rose GA. The Diagnosis of Ischaemic Heart Pain and Intermittent Claudication in Field Surveys. Bull.World Health Organ. 1962; 27:645-58. [PubMed: 13974778]

23. Wills AK, Hardy RJ, Black S, Kuh DJ. Trajectories of Overweight and Body Mass Index in Adulthood and Blood Pressure at Age 53: the 1946 British Birth Cohort Study. J.Hypertens. 2010; 28(4):679-86. [PubMed: 20042875]

24. Schwartz G. Estimating the Dimension of a Model. Annals of Statistics. 1978; 6:461-4. 
25. Little, R.; Rubin, D. Statistical Analysis with Missing Data. John Wiley \& Sons; New York: 1987.

26. Safar ME, Lange C, Tichet J, Blacher J, Eschwege E, Balkau B. The Data From an Epidemiologic Study on the Insulin Resistance Syndrome Study: the Change and the Rate of Change of the AgeBlood Pressure Relationship. J.Hypertens. 2008; 26(10):1903-11. [PubMed: 18806613]

27. Huxley, Rachel; Neil, Andrew; Collins, Rory. Unravelling the Fetal Origins Hypothesis: Is There Really an Inverse Association Between Birthweight and Subsequent Blood Pressure? The Lancet. Aug 31; 2002 360(9334):659-65.

28. Eriksson JG, Forsen TJ, Kajantie E, Osmond C, Barker DJ. Childhood Growth and Hypertension in Later Life. Hypertension. 2007; 49(6):1415-21. [PubMed: 17452506]

29. Blane D, Hart CL, Smith GD, Gillis CR, Hole DJ, Hawthorne VM. Association of Cardiovascular Disease Risk Factors With Socioeconomic Position During Childhood and During Adulthood. The BMJ. Dec 7; 1996 313(7070):1434-8.

30. Li L, Law C, Power C. Body Mass Index Throughout the Life-Course and Blood Pressure in MidAdult Life: a Birth Cohort Study. J.Hypertens. 2007; 25(6):1215-23. [PubMed: 17563534]

31. Hardy R, Kuh D, Langenberg C, Wadsworth ME. Birthweight, Childhood Social Class, and Change in Adult Blood Pressure in the 1946 British Birth Cohort. Lancet. Oct 11; 2003 362(9391): 1178-83. [PubMed: 14568738]

32. Jarvelin MR, Sovio U, King V, Lauren L, Xu B, McCarthy MI, Hartikainen AL, Laitinen J, Zitting P, Rantakallio P, Elliott P. Early Life Factors and Blood Pressure at Age 31 Years in the 1966 Northern Finland Birth Cohort. Hypertension. 2004; 44(6):838-46. [PubMed: 15520301]

33. Law CM, Shiell AW, Newsome CA, Syddall HE, Shinebourne EA, Fayers PM, Martyn CN, de Swiet M. Fetal, Infant, and Childhood Growth and Adult Blood Pressure: a Longitudinal Study From Birth to 22 Years of Age. Circulation. Mar 5; 2002 105(9):1088-92. [PubMed: 11877360]

34. Benetos A, Adamopoulos C, Bureau JM, Temmar M, Labat C, Bean K, Thomas F, Pannier B, Asmar R, Zureik M, Safar M, Guize L. Determinants of Accelerated Progression of Arterial Stiffness in Normotensive Subjects and in Treated Hypertensive Subjects Over a 6-Year Period. Circulation. Mar 12; 2002 105(10):1202-7. [PubMed: 11889014]

35. Bauer DJ, Curran PJ. Distributional Assumptions of Growth Mixture Models: Implications for Overextraction of Latent Trajectory Classes. Psychol.Methods. 2003; 8(3):338-63. [PubMed: 14596495]

36. Heritability of Blood Pressure. Br.Med.J. Jan 21; 1978 1(6106):127-8. [PubMed: 620218] 

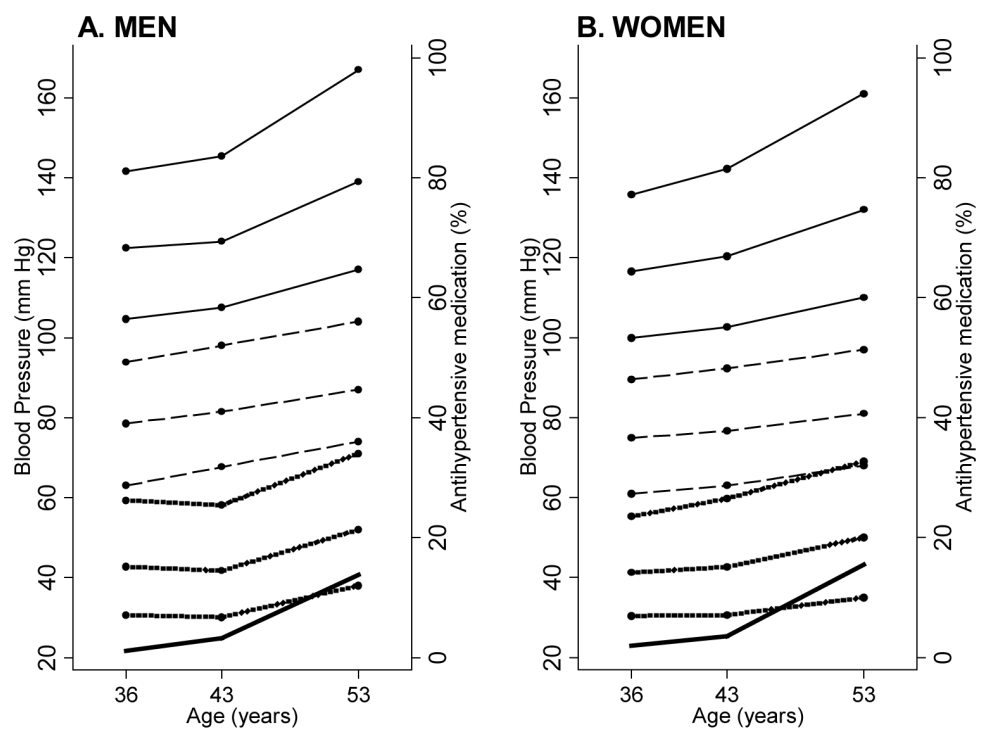

Figure 1.

Median and $10^{\text {th }}$ and $90^{\text {th }}$ centiles for observed systolic BP (top 3 solid lines), diastolic BP (middle broken lines) and pulse pressure (bottom 3 dotted lines) in A. men and B. women at ages 36, 43 and 53 years. The bottom line (thick) is the percentage on treatment for hypertension. . 

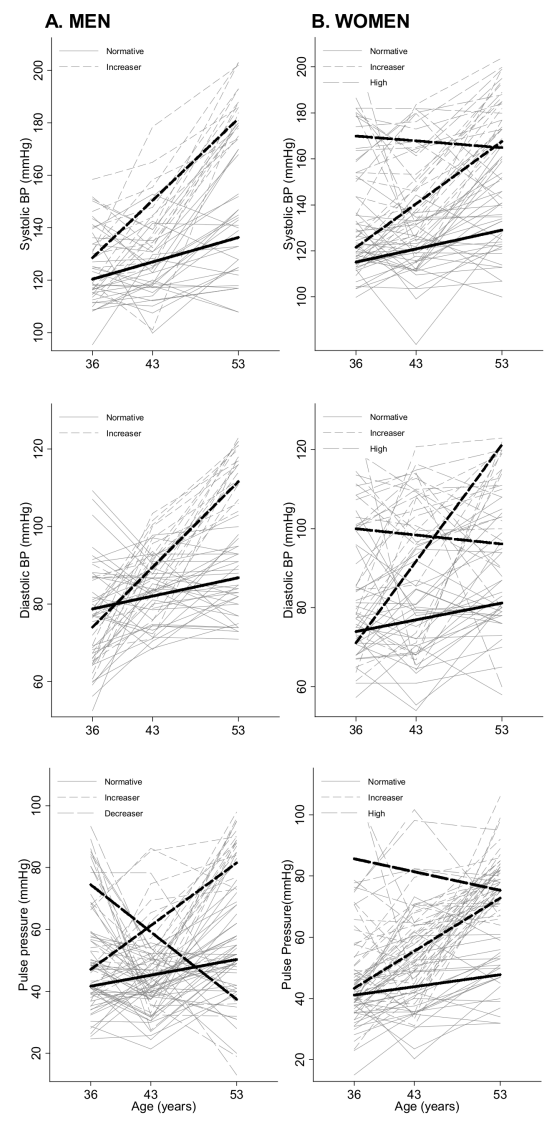

Figure 2.

Predicted mean (thick lines) systolic blood pressure (top panel), diastolic blood pressure (middle panel) and pulse pressure (bottom panel) latent class trajectories from GMM models in A. men and B. women. A random selection of observed individual trajectories stratified by most probable class membership are also plotted (thinner lines). 


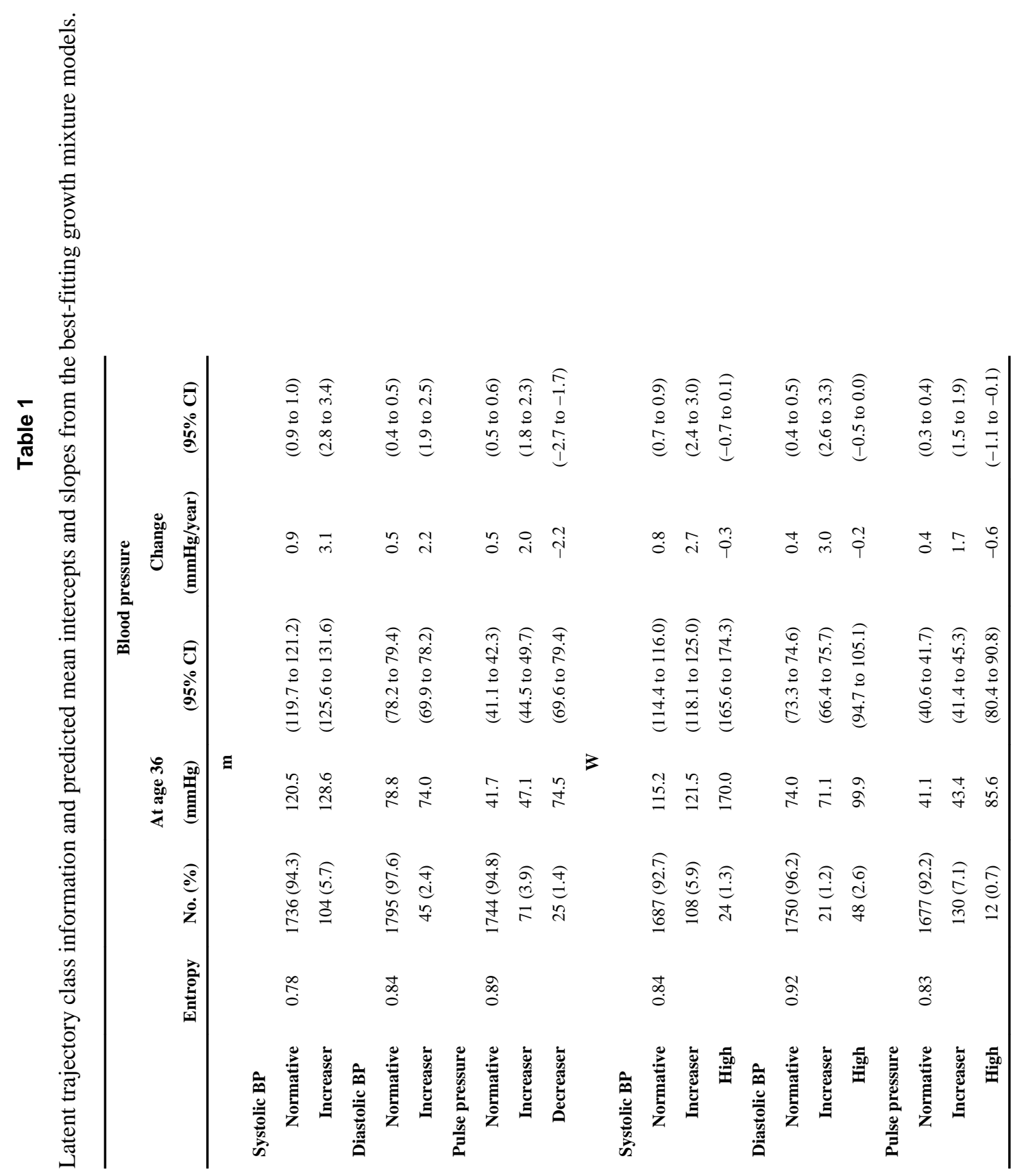


Table 2

Concordance between classes and drivers of pulse pressure: the distribution of systolic and diastolic BPtrajectory class membership within each class of pulse pressure. Row percentages are presented.

\begin{tabular}{|c|c|c|c|c|}
\hline $\begin{array}{r}\text { Systolic BP: } \\
\text { Diastolic BP: }\end{array}$ & $\begin{array}{c}\text { Normal } \\
\text { Normal } \\
\% \text { (95\% CI) }\end{array}$ & $\begin{array}{c}\text { Normal } \\
\text { Increaser/High } \\
\%(95 \% \text { CI })\end{array}$ & $\begin{array}{c}\text { Increaser/High } \\
\text { Normal } \\
\%(95 \% \text { CI })\end{array}$ & $\begin{array}{c}\text { Increaser/High } \\
\text { Increaser/High } \\
\%(95 \% \text { CI })\end{array}$ \\
\hline \multicolumn{5}{|c|}{$\mathrm{m}$} \\
\hline \multicolumn{5}{|l|}{$\begin{array}{l}\text { Pulse } \\
\text { pressure }\end{array}$} \\
\hline \multirow[t]{2}{*}{ Normative } & $(\mathrm{n}=1667)$ & $(\mathrm{n}=19)$ & $(n=41)$ & $(\mathrm{n}=17)$ \\
\hline & 95.6 (94.5 to 96.5$)$ & 1.1 (0.7 to 1.7 ) & 2.4 (1.7 to 3.2$)$ & 0.7 (0.6 to 1.6 ) \\
\hline \multirow[t]{2}{*}{ Increaser } & $(n=27)$ & $(\mathrm{n}=0)$ & $(\mathrm{n}=37)$ & $(\mathrm{n}=7)$ \\
\hline & $38.0(26.8$ to 50.3$)$ & $0(0$ to 5.0$)$ & 52.1 (39.9 to 64.1$)$ & (9.9) \\
\hline \multirow[t]{3}{*}{ Decreaser } & $(\mathrm{n}=22)$ & $(\mathrm{n}=1)$ & $(\mathrm{n}=1)$ & $(n=1)$ \\
\hline & 88.0 (68.9 to 97.4$)$ & $4.0(0.1$ to 20.3$)$ & $4.0(0.1$ to 20.3$)$ & $4.0(0.1$ to 20.3$)$ \\
\hline & & w & & \\
\hline \multicolumn{5}{|l|}{$\begin{array}{l}\text { Pulse } \\
\text { pressure }\end{array}$} \\
\hline \multirow[t]{2}{*}{ Normative } & $(n=1604)$ & $(n=26)$ & $(\mathrm{n}=24)$ & $(\mathrm{n}=23)$ \\
\hline & 95.6 (94.6 to 96.6 ) & 1.6 (1.0 to 2.3 ) & 1.4 (0.9 to 2.1$)$ & $1.4(0.9$ to 2.1$)$ \\
\hline \multirow[t]{2}{*}{ Increaser } & $(\mathrm{n}=54)$ & $(\mathrm{n}=1)$ & $(\mathrm{n}=61)$ & $(n=14)$ \\
\hline & 41.5 (33.0 to 50.5$)$ & 0.8 (0.0 to 4.2 ) & 46.9 (38.1 to 55.9$)$ & $10.8(6.0$ to 17.4$)$ \\
\hline \multirow[t]{2}{*}{ High } & $(n=2)$ & $(\mathrm{n}=0)$ & $(\mathrm{n}=5)$ & $(\mathrm{n}=5)$ \\
\hline & $16.7(2.1$ to 48.4$)$ & $0(0$ to 26.5$)$ & 41.7 (15.2 to 72.3$)$ & $41.7(15.2$ to 72.3$)$ \\
\hline
\end{tabular}

For women only, we pooled the "increaser" and "high" classes because of low cell counts. 


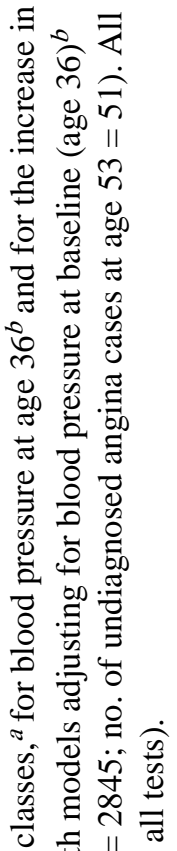

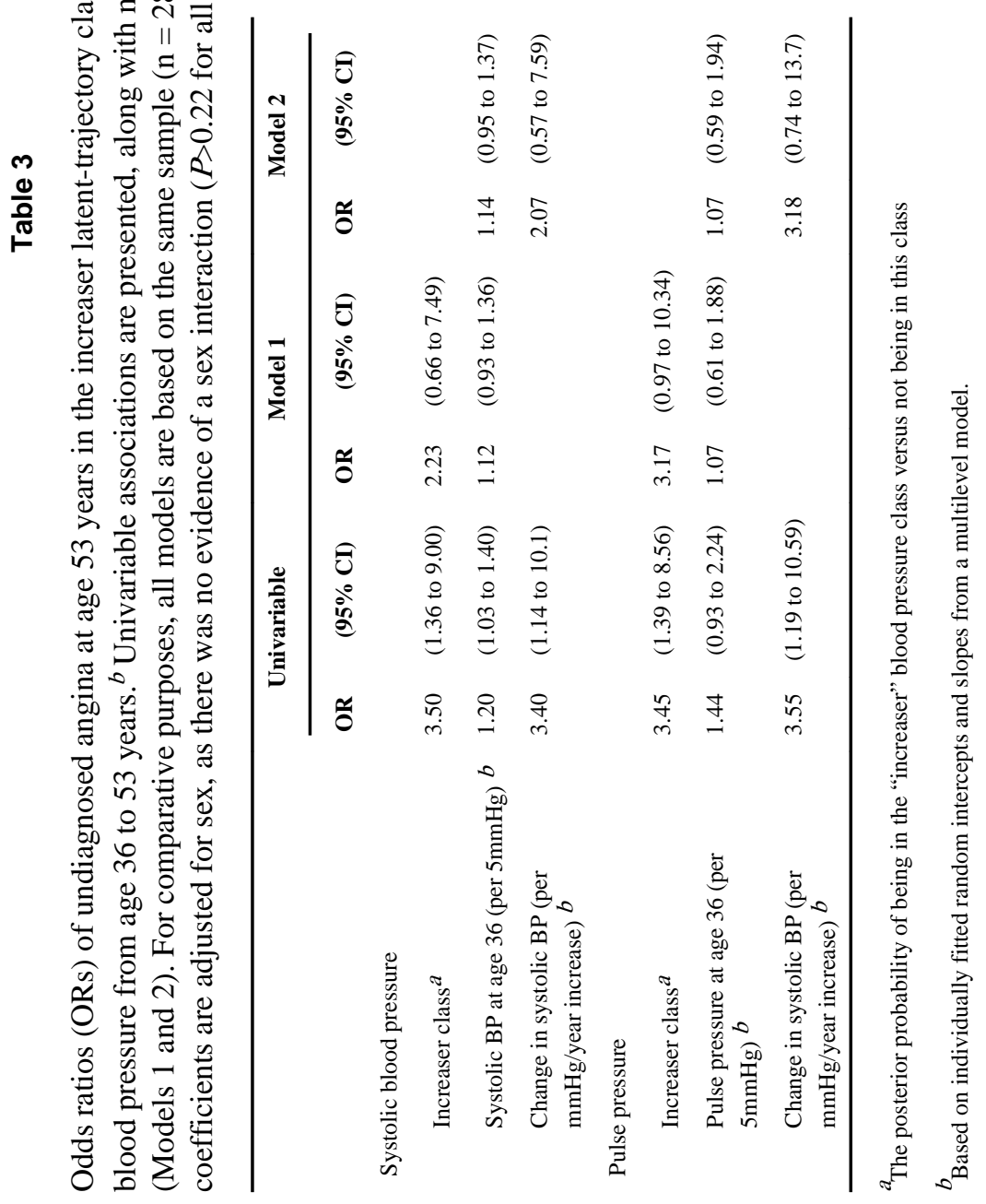

